\title{
Does lack of thoracic trauma attenuate the severity of pulmonary failure? An 8-year analysis of critically injured patients
}

\author{
Xin Huang $^{1} \cdot$ Louis J. Magnotti $^{1} \cdot$ Timothy C. Fabian $^{1} \cdot$ Martin A. Croce ${ }^{1} \cdot$ John P. Sharpe $^{1}$ (])
}

Received: 21 September 2018 / Accepted: 30 January 2019 / Published online: 2 February 2019

c) Springer-Verlag GmbH Germany, part of Springer Nature 2019

\begin{abstract}
Purpose Patients with thoracic trauma are presumed to be at higher risk for pulmonary dysfunction, but adult respiratory distress syndrome (ARDS) may develop in any patient, regardless of associated chest injury. This study evaluated the impact of thoracic trauma and pulmonary failure on outcomes in trauma patients admitted to the intensive-care unit (ICU).

Methods All trauma patients admitted to the ICU over an 8-year period were identified. Patients that died within $48 \mathrm{~h}$ of arrival were excluded. Patients were stratified by baseline characteristics, injury severity, development of ARDS, and infectious complications. Multiple logistic regression was used to determine variables significantly associated with the development of ARDS.

Results 10,362 patients were identified. After exclusions, 4898 (50\%) patients had chest injury and 4975 (50\%) did not. 200 (2\%) patients developed ARDS (3.6\% of patients with chest injury and $0.5 \%$ of patients without chest injury). Patients with ARDS were more likely to have chest injury than those without ARDS (87\% vs $49 \%, p<0.001)$. However, of the patients without chest injury, the development of ARDS still led to a significant increase in mortality compared to those patients without ARDS (58\% vs 5\%, $<<0.001)$. Multiple logistic regression found ventilator-associated pneumonia (VAP) to be the only independent predictor for the development of ARDS in ICU patients without chest injury.

Conclusions ARDS development was more common in patients with thoracic trauma. Nevertheless, the development of ARDS in patients without chest injury was associated with a tenfold higher risk of death. The presence of VAP was found to be the only potentially preventable and treatable risk factor for the development of ARDS in ICU patients without chest injury.
\end{abstract}

Keywords ARDS $\cdot$ Ventilator-associated pneumonia $\cdot$ Trauma $\cdot$ Chest injury

\section{Background}

John P. Sharpe

jsharpe6@uthsc.edu

Xin Huang

Xhuang12@uthsc.edu

Louis J. Magnotti

lmagnott@uthsc.edu

Timothy C. Fabian

tfabian@uthsc.edu

Martin A. Croce

mcroce@uthsc.edu

1 Department of Surgery, University of Tennessee Health Science Center, 910 Madison Ave. \#225, Memphis, TN 38163, USA
Despite advancements in the initial resuscitation, critical care, and ventilator management, the development of acute respiratory distress syndrome (ARDS) is still a challenging problem for the trauma intensivist [1]. Since the initial description of ARDS in 1967, the incidence of this morbidity in the trauma patient appears to be declining, although its associated mortality remains remarkably high, ranging from 7 to $>50 \%$ [2-8] in the current literature.

Patients with thoracic trauma are presumed to be at higher risk for pulmonary dysfunction. However, ARDS may develop in any critically injured patient, regardless of associated chest trauma [8-10]. While it is well known that the pathophysiology and morbidity behind ARDS differs between trauma patients and non-trauma medical patients, this is less understood in trauma patients without chest injury $[3,4,11,12]$. Based on this concern, the purpose 
of this study was to evaluate the impact of thoracic trauma and pulmonary failure on outcomes in trauma patients admitted to the intensive-care unit (ICU).

\section{Methods}

\section{Identification of patients}

Following approval from the Institutional Review Board at the University of Tennessee Health Science Center, consecutive trauma patients requiring ICU admission over an 8-year period were identified from the trauma registry of the Presley Regional Trauma Center in Memphis, Tennessee. This hospital is the only trauma center in Memphis, serves as the only trauma center in the Mid-South area, and is a major teaching facility for the University of Tennessee Health Science Center. The Mid-South is a geographic area of approximately 30,000 square miles surrounding Memphis including western Tennessee, eastern Arkansas, northern Mississippi, and small portions of Missouri and Kentucky. Charts were reviewed for data regarding patient demographics, injury mechanism, severity of injury and shock, infectious morbidity, timing of ARDS, and outcomes. These data were merged with the patient data from the trauma registry (NTRACS version 3.5, Digital Innovations) to compile the database for this study. Ventilator-associated pneumonia (VAP) was recorded as a surrogate for infectious morbidity. All episodes of VAP were diagnosed by bronchoalveolar lavage (BAL) $\left(\geq 10^{5}\right.$ $\mathrm{CFU} / \mathrm{ml}$ in the effluent). Patients that died within $48 \mathrm{~h}$ of presentation were excluded.

\section{Chest injury}

The chest Abbreviated Injury Scale (chest-AIS) was used to differentiate between patients with and without chest injury. Patients without thoracic trauma were defined as having a chest-AIS of 0 . Patients with thoracic trauma were defined as having a chest-AIS greater than or equal to 1 .

\section{Outcomes}

The primary outcome of interest was pulmonary failure, defined as the development of ARDS. The diagnosis of ARDS required $\mathrm{PaO}_{2} / \mathrm{FiO}_{2}$ ratio $<200$, bilateral infiltrate on chest radiography, and no evidence of congestive heart failure. Secondary outcomes included hospital and intensive-care unit (ICU) length of stay, ventilator days, and inhospital mortality.

\section{Comparison}

Characteristics and outcomes were compared between the patients who developed ARDS and those who did not develop ARDS. This analysis was then repeated for those patients without chest injury. Statistical analyses were performed using a Student's $t$ test and Chi-square or Fisher's exact test where appropriate. A Kolmogorov-Smirnov Test for normality was performed for each continuous variable. If the Kolmogorov-Smirnov Test provided a statistic with a $p$ value $>0.05$, then the data were considered normally distributed. When the data were not normally distributed, a Wilcoxon rank sum test was performed for these continuous variables. Multivariable logistic regression was performed to determine variables significantly associated with the development of ARDS in the study population. Variables that had a $p$ value $<0.2$ on the initial univariable analysis were chosen as prospective covariates. The final multivariable model was constructed in a backward stepwise fashion to identify independent predictors for the development of ARDS. A $p$ value of $<0.05$ was considered statistically significant.

\section{Results}

\section{Study population}

During the 8-year study period (ending October 2016), 10,362 patients were admitted to the ICU at the Presley Regional Trauma Center. 489 patients died within $48 \mathrm{~h}$ of arrival to the hospital, leaving 9873 patients that met inclusion criteria for the study. The majority of patients were male $(73 \%)$. These patients ranged in age from 13 to 100 years (mean 43 years), with a mean injury severity score (ISS) and Glasgow coma scale score (GCS) of 21 and 13, respectively. Chest injuries occurred in 4898 patients (50\%) and 200 patients (2\%) suffered ARDS. Overall mortality was $6.2 \%$.

\section{Adult respiratory distress syndrome}

Table 1 demonstrates a comparison of characteristics between patients with ARDS and those without ARDS. The groups were similar with respect to age, gender, and mechanism. However, patients with ARDS had a greater degree of shock on presentation compared to the patients without ARDS, as demonstrated by a larger RBC transfusion requirement ( 9 vs 5 units, $p<0.001$ ) and both a lower admission systolic blood pressure (125 vs $133, p=0.002)$ and base excess $(-6.0$ vs $-3.7, p<0.001)$. Patients with ARDS were also more severely injured, as shown by a 
Table 1 Comparison of patient characteristics and outcomes between patients with ARDS and those without ARDS

\begin{tabular}{lllr}
\hline & ARDS & No ARDS & $p$ \\
\hline$n$ & 200 & 9673 & \\
Age & $45(16)$ & $43(19)$ & 0.07 \\
Male & $144(72 \%)$ & $6964(72 \%)$ & 0.71 \\
Blunt mechanism & $162(81 \%)$ & $7738(80 \%)$ & 0.79 \\
Base excess & $-6.0(5.6)$ & $-3.7(5.3)$ & $<0.001$ \\
RBC transfusion (units) & $9(4,17)$ & $5(2,9)$ & $<0.001$ \\
Systolic blood pressure & $125(34)$ & $133(29)$ & 0.002 \\
Glasgow coma scale & $12(3,15)$ & $15(12,15)$ & $<0.001$ \\
Injury severity score & $33(25,31)$ & $19(13,29)$ & $<0.001$ \\
Chest injury & $174(87 \%)$ & $4740(49 \%)$ & $<0.001$ \\
Ventilator-associated pneumonia & $100(50 \%)$ & $1064(11 \%)$ & $<0.001$ \\
Ventilator days & $14.5(8,24)$ & $1(0,5)$ & $<0.001$ \\
Hospital length of stay (days) & $25(12,46)$ & $10(5,21)$ & $<0.001$ \\
Intensive-care unit length of stay (days) & $17.5(9,32)$ & $4(2,10)$ & $<0.001$ \\
Mortality & $80(40 \%)$ & $532(5.5 \%)$ & $<0.001$ \\
\hline
\end{tabular}

Categorical variables are presented as total number with percentage in parentheses; normally distributed continuous variables are presented as means with standard deviations in parentheses; non-normally distributed continuous variables are presented as medians with interquartile ranges in parentheses higher injury severity score (33 vs $19, p<0.001)$ and a lower GCS (12 vs 15, $p<0.001)$. Notably, patients with ARDS were more likely to have chest injury than those without ARDS (87\% vs $49 \%, p<0.001)$.

Table 1 also demonstrates a comparison of outcomes between patients with ARDS and those without ARDS. Patients with ARDS had a longer hospital (25 vs 10 days, $p<0.001)$ and ICU length of stay (17.5 vs 4 days, $p<0.001)$, required more time on the ventilator (14.5 vs 1 days, $p<0.001$ ), and had a higher mortality (40\% vs $5.5 \%$, $p<0.001)$ compared to patients without ARDS.

\section{ARDS and chest injury}

To better understand to relationship between thoracic trauma and ARDS, of the patients with ARDS, those with chest injury were compared to those without chest injury (Table 2). The two groups were similar with respect to most variables. However, despite being less severely injured
Table 2 Comparison of patient characteristics and outcomes between ARDS patients with chest injury and ARDS patients without chest injury

\begin{tabular}{lllc}
\hline & Chest injury & No chest injury & $p$ \\
\hline$n$ & 174 & 26 & \\
Age & $46(17)$ & $40(13)$ & 0.10 \\
Male & $122(70 \%)$ & $21(81 \%)$ & 0.35 \\
Blunt & $143(82 \%)$ & $19(73 \%)$ & 0.29 \\
Base excess & $-6.3(5.5)$ & $-4.0(6.5)$ & 0.09 \\
RBC transfusion (units) & $9(4,18)$ & $9(6,14)$ & 0.91 \\
Systolic blood pressure & $124(35)$ & $129(27)$ & 0.57 \\
Glasgow coma scale & $12(3,15)$ & $14(7,15)$ & 0.37 \\
Injury severity score & $34(27,41)$ & $18.5(16,26)$ & $<0.001$ \\
Ventilator-associated pneumonia & $87(50 \%)$ & $14(54 \%)$ & 0.71 \\
Ventilator days & $14(7,23)$ & $17(9,40)$ & 0.10 \\
Hospital length of stay (days) & $24(12,41)$ & $26.5(14,54)$ & 0.41 \\
Intensive-care unit length of stay (days) & $17(9,31)$ & $20.5(10,45)$ & 0.16 \\
Mortality & $64(37 \%)$ & $15(58 \%)$ & 0.04 \\
\hline
\end{tabular}

Categorical variables are presented as total number with percentage in parentheses; normally distributed continuous variables are presented as means with standard deviations in parentheses; non-normally distributed continuous variables are presented as medians with interquartile ranges in parentheses 
(ISS, 18.5 vs $34, p<0.001$ ), ARDS patients without chest injury suffered a much higher mortality compared to ARDS patients with chest injury (58\% vs $37 \%, p=0.04)$.

\section{Patients without chest injury}

To evaluate the impact of ARDS on patients without thoracic trauma, patients with ARDS were compared to those patients without ARDS within the subgroup of patients without chest injury (Table 3 ). The groups were similar with respect to almost all characteristics. However, of the patients without chest injury, the incidence of VAP was much higher in those patients with ARDS than those patients without ARDS $(54 \%$ vs $8 \%, p<0.001)$. Furthermore, the patients with ARDS had a larger RBC transfusion requirement (9 vs 4 units, $p<0.001$ ) and had a higher injury severity score (18.5 vs $16, p=0.01)$ compared to patients without ARDS. Notably, all of the cases of VAP for patients without thoracic trauma occurred before the development of ARDS.

Table 3 also demonstrates a comparison of outcomes between patients with ARDS and those without ARDS within the subgroup of patients without chest injury. Again, patients with ARDS had a longer hospital (26.5 vs 8 days, $p<0.001)$ and ICU length of stay (20.5 vs 3 days, $p<0.001$ ), and required more time on the ventilator (17 vs 0 days, $p<0.001)$. Notably, of the patients without chest injury, the development of ARDS still led to an 11-fold increase in mortality compared to those patients without ARDS (58\% vs $5 \%, p<0.001)$.

\section{Multivariable logistic regression}

A multivariable logistic regression model was then developed to identify variables significantly associated with ARDS in the total study population after adjusting for potential covariates. After adjusting for severity of the initial shock (RBC transfusion and admission base excess and systolic blood pressure), severity of injury (chest-AIS and admission GCS) and VAP, only RBC transfusion, chest-AIS, and VAP were found to be significantly associated with the development of ARDS in these patients (Table 4). Stepwise multivariable logistic regression analysis found VAP, RBC transfusion, chest-AIS, and admission GCS to be the only independent predictors of ARDS in the study population.

A multivariable logistic regression model was then developed to identify variables significantly associated with ARDS in patients with chest injury after adjusting for potential covariates (Table 4). After adjusting for severity of initial shock (RBC transfusion and admission base excess and systolic blood pressure), severity of injury (chest-AIS and admission GCS), and VAP, only RBC transfusion and VAP were found to be significantly associated with the development of ARDS in these patients (Table 4). However, stepwise multivariable logistic regression analysis found VAP, RBC transfusion, chest-AIS, and admission GCS to be the only independent predictors of ARDS in the study population.

A multivariable logistic regression model was then developed to identify variables significantly associated with ARDS in patients without chest injury after adjusting for potential covariates. After adjusting for severity of the initial shock (RBC transfusion and admission base excess and
Table 3 Comparison of patient characteristics and outcomes between non-thoracic injured patients with ARDS and those without ARDS

\begin{tabular}{lllc}
\hline & ARDS & No ARDS & $p$ \\
\hline$n$ & 26 & 4949 & \\
Age & $40(13)$ & $43(19)$ & 0.31 \\
Male & $21(81 \%)$ & $3662(74 \%)$ & 0.65 \\
Blunt mechanism & $19(73 \%)$ & $3712(75 \%)$ & 0.45 \\
Base excess & $-4.0(6.5)$ & $-3.1(5.3)$ & 0.53 \\
RBC transfusion (units) & $9(6,14)$ & $4(2,9)$ & $<0.001$ \\
Systolic blood pressure & $129(27$ & $136(29)$ & 0.24 \\
Glasgow coma scale & $14(8,15)$ & $15(12,15)$ & 0.09 \\
Injury severity score & $18.5(16,26)$ & $16(9,21)$ & 0.01 \\
Ventilator-associated pneumonia & $14(54 \%)$ & $396(8 \%)$ & $<0.001$ \\
Ventilator days & $17(9,40)$ & $0(0,3)$ & $<0.001$ \\
Hospital length of stay (days) & $26.5(14,54)$ & $8(4,17)$ & $<0.001$ \\
Intensive-care unit length of stay (days) & $20.5(10,45)$ & $3(2,7)$ & $<0.001$ \\
Mortality & $15(58 \%)$ & $247(5 \%)$ & $<0.001$ \\
\hline
\end{tabular}

Categorical variables are presented as total number with percentage in parentheses; normally distributed continuous variables are presented as means with standard deviations in parentheses; non-normally distributed continuous variables are presented as medians with interquartile ranges in parentheses 
Table 4 Adjusted odds ratios for the development of ARDS

\begin{tabular}{lll}
\hline & Adjusted OR & $95 \%$ CI \\
\hline All patients ( $n=9873)$ & & \\
Admission base excess & 0.98 & $0.95-1.01$ \\
Admission systolic blood pressure & 1.00 & $0.99-1.01$ \\
RBC transfusion (units) & 1.02 & $1.01-1.03$ \\
Glasgow coma scale & 0.97 & $0.94-1.01$ \\
Chest abbreviated injury scale & 1.21 & $1.01-1.46$ \\
Ventilator-associated pneumonia & 2.68 & $1.87-3.84$ \\
Chest injury (n=4898) & & \\
Admission base excess & 0.98 & $0.95-1.01$ \\
Admission systolic blood pressure & 1.00 & $0.99-1.01$ \\
RBC transfusion (units) & 1.03 & $1.01-1.04$ \\
Glasgow coma scale & 0.97 & $0.94-1.01$ \\
Chest abbreviated injury scale & 1.20 & $0.99-1.45$ \\
Ventilator-associated pneumonia & 2.64 & $1.81-3.84$ \\
No chest injury ( $n=4975$ ) & & \\
Admission base excess & 0.99 & $0.90-1.08$ \\
Admission systolic blood pressure & 0.99 & $0.98-1.01$ \\
RBC transfusion (units) & 1.00 & $0.98-1.03$ \\
Glasgow coma scale & 1.00 & $0.90-1.12$ \\
Injury severity score & 0.98 & $0.93-1.04$ \\
Ventilator-associated Pneumonia & 13.3 & $4.14-43.1$ \\
\hline
\end{tabular}

systolic blood pressure), severity of injury (chest-AIS and admission GCS), and VAP, only VAP was found to be significantly associated with the development of ARDS in these patients (Table 4). In fact, stepwise multivariable logistic regression analysis found VAP to be the only independent predictor of ARDS in patient without chest injuries.

\section{Discussion}

In this series of 9873 trauma patients admitted to the ICU, the development of moderate-to-severe ARDS was relatively uncommon with an incidence of $2 \%$. This is much lower compared to the $7.3 \%$ seen in general ICU admissions [13] or the $12-25 \%$ of trauma patients reported by the previous studies $[8,14,15]$. Despite lower occurrence, the trauma patients who developed moderate-to-severe ARDS had a mortality of $40 \%$, which is similar to what is reported by the other studies [2-8]. The reduction in ARDS occurrence may speak well for the improvement in patient management and prevention, but the unchanging mortality demonstrates that, rather, little advancement has been made in treating this condition.

Trauma-associated ARDS has been shown to be different, both clinically and biologically, from that caused by the other clinical disorders [16]. Given this fact, there could be distinct forms of ARDS even within the post-traumatic population [3]. Multiple studies have revealed lower mortality rates in trauma patients $[4,16$, 17], but the mortality variation of ARDS within different trauma patients has yet to be examined in a large-sample population prior to this study.

Thoracic trauma is a well-known risk factor for ARDS. The inflammatory response to a unilateral insult can progress into bilateral pulmonary dysfunction if the dysregulated inflammation is of sufficient magnitude [18]. In 1999, Croce et al. evaluated 178 patients with post-traumatic ARDS and determined that there were two forms of pulmonary failure based on time to diagnosis [3]. The early ARDS (within $48 \mathrm{~h}$ after admission) was characterized by hemorrhagic shock with capillary leak. Late ARDS ( $>48 \mathrm{~h}$ between admission and diagnosis) frequently followed pneumonia. Similar to the late ARDS group studied by Croce et al., mean time to diagnosis of ARDS was 6.2 days for those patients in the current study without chest injury. Furthermore, the development of VAP was also identified as an independent predictor of ARDS for this subgroup.

Although the majority of the patients that developed moderate-to-severe ARDS in the current study presented with chest injury on admission, a subset (13\%) of ARDS patients did not sustain any thoracic trauma. Despite being less severely injured, patients without chest injury had significantly higher mortality compared to those patients with chest injury. As previously stated, VAP was found to be the only independent factor associated with the development of ARDS in patients without chest injury. However, the incidence of VAP in ARDS patients without chest injury was not significantly different from that of ARDS patients with chest injury ( $54 \%$ vs. 50\%, $p=0.71$ ), and, therefore, cannot account for the mortality difference between the two groups. These results suggest that VAP may play a role in the development of ARDS in trauma patients without thoracic injury, but it does not appear to be a contributor to the mortality difference between those ARDS patients with and without chest injury.

The presence of VAP was found to be the only potentially preventable and treatable risk factor for the development of ARDS in ICU patients without chest injury. Preventing the development of VAP in a critically injured patient is easier said than done. In 2005, the Institution for Healthcare Improvement introduced the concept of a ventilator bundle to improve the outcomes of mechanical ventilation and, thus, reduce VAP. The ventilator bundle consists of stress ulcer prophylaxis, deep venous thrombosis prophylaxis, headof-bed elevation, and daily sedation vacation with weaning assessment. However, in 2013, a prospective observational multi-institutional study found compliance with the ventilator bundle was not associated with VAP prevention. Nevertheless, over the last 2 decades, many advancements have been made in protocol-driven pathways for the early 
diagnosis and appropriate treatment of VAP that may serve to limit further pulmonary complications such as ARDS [19-21].

In the current study, a greater degree of shock, worse neurologic status, and more severe injury were observed in all trauma patients that developed moderate-to-severe ARDS compared to those who did not. Although these differences were no longer significant when trauma patients without thoracic trauma were examined, mortality was worsened by more than 11-fold. This finding further reinforces that ARDS is a different entity in trauma patients without chest injury. Although lacking thoracic injury may very well reduce the risk of developing ARDS, it does not attenuate the severity. In fact, the trauma patients without thoracic trauma seem to have worse prognosis if ARDS were to develop.

The major limitations of this study include, first, the retrospective design. This precludes the exclusion of selection bias and unevaluated differences as potential confounding variables. In addition, this allows only for associations to be made, and cannot account for all potential confounding differences. Furthermore, the data points are limited by what can be collected through available records. Data regarding specific treatments or detailed ventilator settings that could affect mortality were not examined. In addition, patients with thoracic trauma were defined as having a chest-AIS greater than or equal to 1 . Including patients with lower chest-AIS values may potentially over-simplify the cohorts. However, we did not want to risk including any patients with thoracic trauma in the non-chest injury group. Therefore, we felt this to be the purest method. Finally, the development of ARDS was not separated into mild, moderate, and severe categories as defined by the Berlin definition [22]. Approximately half of the patients in the study were admitted prior to the publishing of the Berlin definition, when the American-European Consensus Conference criteria [23] were used for ARDS. The diagnosis coding in the trauma registry only allowed for the identification for the patients with moderate-to-severe ARDS.

\section{Conclusion}

In summary, lack of thoracic injury does not attenuate the severity of ARDS in trauma patients. Although ARDS is more commonly observed in those who sustain injury to the chest, the development of ARDS in patients without chest injury is associated with a worse prognosis. Unlike ARDS in setting of chest injury, VAP is the only significant risk factor associated with ARDS in trauma patients without chest injury. Future studies are needed to examine this population in closer detail to develop appropriate management protocols.
Author contributions Study conception and design: Huang and Sharpe. Acquisition of data: Sharpe and Huang. Analysis and interpretation of data: Sharpe, Huang, Magnotti, Fabian, and Croce. Drafting of manuscript: Sharpe and Huang. Critical revision: Magnotti, Fabian, and Croce.

\section{Compliance with ethical standards}

Conflict of interest Xin Huang MD does not have any financial disclosures or conflicts of interest to declare. Louis J Magnotti MD does not have any financial disclosures or conflicts of interest to declare. Timothy C Fabian MD does not have any financial disclosures or conflicts of interest to declare. Martin A Croce MD does not have any financial disclosures or conflicts of interest to declare. John P Sharpe MD, MS does not have any financial disclosures or conflicts of interest to declare.

\section{References}

1. Ashbaugh DG, Bigelow DB, Petty TL, Levine BE. Acute respiratory distress in adults. Lancet. 1967;2:319-23.

2. Fahr M, Jones G, O'Neal H, Duchesne J, Tatum D. Acute respiratory distress syndrome incidence, but not mortality, has decreased nationwide: a national trauma data bank study. Am Surg. 2017;83(4):323-31.

3. Croce MA, Fabian TC, Davis KA, Gavin TJ. Early and late acute respiratory distress syndrome: two distinct clinical entities. J Trauma. 1999;46(3):361-6 (discussion 366-8).

4. Milberg JA, Davis DR, Steinberg KP, Hudson LD. Improved survival of patients with acute respiratory distress syndrome (ARDS): 1983-1993. JAMA. 1995;273:306-9.

5. Eberhard LW, Morabito DJ, Matthay MA, Mackersie RC, Campbell AR, Marks JD, Alonso JA, Pittet JF. Initial severity of metabolic acidosis predicts the development of acute lung injury in severely traumatized patients. Crit Care Med. 2000;28:125-31.

6. Salim A, Martin M, Constantinou C, Sangthong B, Brown C, Kasotakis G, Demetriades D, Belzberg H. Acute respiratory distress syndrome in the trauma intensive care unit: morbid but not mortal. Arch Surg. 2006;141:655-8.

7. Haider AH, Gupta S, Zogg CK, Kisat MT, Schupper A, Efron DT, Haut ER, Obirieze AC, Schneider EB, Pronvost PJ, et al. Beyond incidence: costs of complications in trauma and what it means for those who pay. Surgery. 2015;158:96-103.

8. Treggiari MM, Hudson LD, Martin DP, Weiss NS, Caldwell E, Rubenfeld G. Effect of acute lung injury and acute respiratory distress syndrome on outcome in critically ill trauma patients. Crit Care Med. 2004;32:327-31.

9. Miller PR, Croce MA, Bee TK, Qaisi WG, Smith CP, Collins GL, Fabian TC. ARDS after pulmonary contusion: accurate measurement of contusion volume identifies high-risk patients. J Trauma. 2001;51(2):223-8 (discussion 229-30).

10. Miller PR, Croce MA, Kilgo PD, Scott J, Fabian TC. Acute respiratory distress syndrome in blunt trauma: identification of independent risk factors. Am Surg. 2002;68(10):845-50 (discussion 850-1).

11. Cooke CR, Kahn JM, Caldwell E, Okamoto VN, Heckbert SR, Hudson LD, Rubenfeld GD. Predictors of hospital mortality in a population-based cohort of patients with acute lung injury. Crit Care Med. 2008;36(5):1412-20.

12. Luo L, Shaver CM, Zhao Z, Koyama T, Calfee CS, Bastarache JA, Ware LB. Clinical predictors of hospital mortality differ between direct and indirect ARDS. Chest. 2017;151(4):755-63.

13. Bellani G, Laffey JG, Pham T, Fan E, Brochard L, Esteban A, Gattinoni L, van Haren F, Larsson A, McAuley DF, et al. 
Epidemiology, pattern of care, and mortality for patients with acute respiratory distress syndrome in intensive care units in 50 countries. JAMA. 2016;315(8):788-800.

14. Poole GV, Ward EF, Griswold JA, Muakkassa FF, Hsu HS. Complications of pelvic fractures from blunt trauma. Am Surg. 1992;58:225-31.

15. Rubenfeld GD, Caldwell E, Peabody E, Weaver J, Martin DP, Neff $\mathrm{M}$, Stern EJ, Hudson LD. Incidence and outcomes of acute lung injury. N Engl J Med. 2005;353:1685-93.

16. Calfee CS, Eisner MD, Ware LB, Thompson BT, Parsons PE, Wheeler AP, Korpak A, Matthay MA. Trauma-associated lung injury differs clinically and biologically from acute lung injury due to other clinical disorders. Crit Care Med. 2007;35(10):2243-50.

17. DiRusso SM, Nelson LD, Safcsak K, Miller RS. Survival in patients with severe adult respiratory distress syndrome treated with high-level positive end-expiratory pressure. Crit Care Med. 1995;23:1485-96.

18. Sutyak JP, Wohltmann CD, Larson J. Pulmonary contusions and critical care management in thoracic trauma. Thorac Surg Clin. 2007;17(1):11-23.

19. Croce MA, Fabian TC, Schurr MJ, Boscarino R, Pritchard FE, Minard G, Patton JH Jr, Kudsk KA. Using bronchoalveolar lavage to distinguish nosocomial pneumonia from systemic inflammatory response syndrome: a prospective analysis. J Trauma. 1995;39(6):1134-9 (discussion 1139-40).

20. Magnotti LJ, Schroeppel TJ, Fabian TC, Clement LP, Swanson JM, Fischer PE, Bee TK, Maish GO 3rd, Minard G, Zarzaur BL, Croce MA. Reduction in inadequate empiric antibiotic therapy for ventilator-associated pneumonia: impact of a unit-specific treatment pathway. Am Surg. 2008;74(6):516-22 (discussion 522-3).

21. Sharpe JP, Magnotti LJ, Weinberg JA, Swanson JM, Wood GC, Fabian TC, Croce MA. Impact of pathogen-directed antimicrobial therapy for ventilator-associated pneumonia in trauma patients on charges and recurrence. J Am Coll Surg. 2015;220(4):489-95.

22. ARDS Definition Task Force. Ranieri VM, Rubenfeld GD, Thompson BT, Ferguson ND, Caldwell E, Fan E, Camporota L, Slutsky AS. Acute respiratory distress syndrome: the Berlin Definition. JAMA 2012; 307(23):2526-33.

23. Bernard GR, Artigas A, Brigham KL, Carlet J, Falke K, Hudson L, Lamy M, Legall JR, Morris A, Spragg R, et al: The AmericanEuropean Consensus Conference on ARDS: definitions, mechanisms, relevant outcomes, and clinical trial coordination. Am J Respir Crit Care Med. 1994; 149(3 pt 1):818-824. 